\title{
The Effect of Open-Cell Metal Foams Strut Shape on Convection Heat Transfer and Pressure Drop
}

\author{
Giuseppe Ambrosio*, Nicola Bianco*\#, Wilson K. S. Chiu ${ }^{\circ}$, Marcello Iasiello*, \\ Vincenzo Naso*, Maria Oliviero $^{\text {}}$ \\ (*) DII - Università degli studi Federico II - P.le Tecchio, 80 - Napoli - 80125 - Italy \\ $\left(^{\circ}\right)$ University of Connecticut - Storrs (CT) - USA \\ $\left({ }^{\circledR}\right)$ Consiglio Nazionale delle Ricerche - P.le Fermi, 1 - Portici (NA) - 80055 - Italy
}

\begin{abstract}
Metal foams are promising materials for applications where enhancement of heat transfer is needed. Their performance depends on morphological parameters, such as the diameters of cells, pores, and struts, the porosity, the shape of struts.

The effect of strut shape on convection heat transfer and pressure drop in open-cell metal foams has been investigated numerically in this paper by introducing the foam shape factor, a parameter that characterizes the shape of the strut. The analysis has been carried out both on real and ideal foams. The geometry of three 40 Pores Per Inch (PPI) real foams, with 0.87, 0.94 and 0.96 average measured porosities, was determined with X-ray Computed Microtomography (XCT) and a morphological analysis of the XCT data was carried out. The geometry of the ideal foams, based on the Lord Kelvin foam model, with the same PPI and porosities as those of the real foams, was generated with the free-to-use software Surface Evolver, building up foams with different strut shapes. Governing equations have been solved with a finite element scheme, assuming a uniform heat flux condition at the solid/fluid boundary of the foam.

Results are presented in terms of convection heat transfer coefficients and pressure distributions as a function of strut shape parameters. The comparison between predictions for ideal and real foams, for different strut shapes, confirms that, in all cases, the closer the form of the ideal strut to that of the real strut the better the agreement among predictions. It is also pointed out that the convection heat transfer coefficient is maximized when, at equal porosity, the ligament shape is convex, while a concave strut shape maximizes the pressure drop.
\end{abstract}

Keywords: Metal foams, Convection heat transfer, Pressure drop, Strut shape effect.

\# Corresponding author: nicola.bianco@ unina.it 


\begin{tabular}{|c|c|c|c|c|}
\hline \multicolumn{5}{|c|}{ Nomenclature } \\
\hline$C$ & form coefficient $(1 / \mathrm{m})$ & $\beta$ & \multicolumn{2}{|l|}{ foam shape factor } \\
\hline$C V$ & coefficient of variation & $\varepsilon$ & \multicolumn{2}{|l|}{ porosity } \\
\hline$d$ & diameter $(m)$ & $\lambda$ & \multicolumn{2}{|c|}{ thermal conductivity (W/m K) } \\
\hline$h_{c}$ & heat transfer coefficient $\left(\mathrm{W} / \mathrm{m}^{2} \mathrm{~K}\right)$ & $\mu$ & \multicolumn{2}{|c|}{ dynamic viscosity (kg/m s) } \\
\hline$h_{v}$ & volumetric heat transfer coefficient $\left(\mathrm{W} / \mathrm{m}^{3} \mathrm{~K}\right)$ & $v$ & \multirow{2}{*}{\multicolumn{2}{|c|}{$\begin{array}{l}\text { kinematic viscosity }\left(\mathrm{m}^{2} / \mathrm{s}\right) \\
\text { density }\left(\mathrm{kg} / \mathrm{m}^{3}\right)\end{array}$}} \\
\hline$K$ & permeability $\left(\mathrm{m}^{2}\right)$ & $\rho$ & & \\
\hline$L$ & length $(\mathrm{m})$ & $\Omega$ & \multicolumn{2}{|c|}{ cross section area $\left(\mathrm{m}^{2}\right)$} \\
\hline$n$ & normal vector & \multicolumn{3}{|c|}{ Subscripts } \\
\hline $\mathrm{Nu}$ & Nusselt number & 0 & \multicolumn{2}{|l|}{ ambient, inlet } \\
\hline$p$ & pressure $(\mathrm{Pa})$ & $c$ & \multicolumn{2}{|l|}{ cell } \\
\hline PPI & pores per inch $(0.0254 / \mathrm{m})$ & $\operatorname{circ}$ & \multicolumn{2}{|l|}{ circle } \\
\hline$q$ & heat flux $\left(\mathrm{W} / \mathrm{m}^{2}\right)$ & dom & \multicolumn{2}{|l|}{ computational domain } \\
\hline $\operatorname{Re}$ & Reynolds number & $f$ & \multicolumn{2}{|l|}{ fluid } \\
\hline$S$ & surface area $(m)$ & $h$ & \multicolumn{2}{|l|}{ hydraulic } \\
\hline$t$ & strut tapering factor & $j$ & \multicolumn{2}{|l|}{ junction } \\
\hline$T$ & temperature $(\mathrm{K})$ & $p$ & \multicolumn{2}{|l|}{ pore } \\
\hline $\boldsymbol{u}$ & velocity vector $(\mathrm{m} / \mathrm{s})$ & $R V E$ & \multicolumn{2}{|l|}{$\begin{array}{l}\text { Represent at ive } \\
\text { El ement }\end{array}$} \\
\hline$V$ & total volume of the foam $\left(\mathrm{m}^{3}\right)$ & $S$ & \multicolumn{2}{|l|}{ surface-fluid interface } \\
\hline$x, y, z$ & rectangular coordinates $(\mathrm{m})$ & $s$ & \multicolumn{2}{|l|}{ strut } \\
\hline \multicolumn{2}{|c|}{ Greek letters } & Other & & \\
\hline$\alpha$ & thermal diffusivity $\left(\mathrm{m}^{2} / \mathrm{s}\right)$ & & \multicolumn{2}{|l|}{ average } \\
\hline
\end{tabular}

\section{Introduction}

Open-cell metal foams are promising materials for applications where enhancing the heat transfer is a primary task. Examples of applications are compact heat exchangers $[1,2]$, heat sinks, volumetric solar receivers, and burners. The advantages of foams are tortuosity, that favors flow mixing and turbulence, low weight, effective thermal conductivity and high heat transfer surface to volume ratio. However, these characteristics enhance convection heat transfer, but also increase pressure drop.

The temperature field in open cell foams can be investigated experimentally, but the complicate structure make accurate measurements difficult. Numerical or analytical predictions can be obtained by solving the appropriate volume-averaged governing equations, accounting for both phases of the porous medium in an unique equivalent continuous medium. The temperature field can be described with a Local Thermal Equilibrium (LTE) model, assuming fluid and solid phases to be in thermal equilibrium, or with a Local Thermal Non-Equilibrium (LTNE) model, that takes into account convection heat transfer between the two phases by a volumetric heat transfer coefficient. The validity of the LTE model was tested by Amiri and Vafai [3].

When governing equations for fluid flow and heat transfer are written with the VolumeAveraging Technique (VAT), closing coefficients, like the aforementioned volumetric heat transfer coefficient, the permeability and the inertial coefficient, need to be known in order to 
solve the governing equations. The last two closing coefficients mentioned above are constant quantities that describe pressure drop in a porous medium. Three different methods are used to obtain them. The first method is based on experimental measurements. Studies on pressure drop and convection heat transfer for different open-cell metal foams were experimentally performed by Mancin et al. [4-6]. The second method is based on numerical simulations on foam morphologies obtained by a tomographic scan of a real foam sample [7]. It was also used by Ranut et al. [8], who investigated the effect of pores per inch (PPI) and anisotropy on heat transfer coefficients and pressure drops. Zafari et al. [9] obtained a correlation for Nusselt number, which takes into account the effect of porosity. Numerical simulations were also performed by Diani et al. [10] on copper foams with different PPIs. The third method is based on a computationallygenerated model of the foam. Many foam geometries have been proposed. Lu et al. [11] used a cubic unit cell, made by heated slender cylinders, to investigate convection heat transfer. Kelvinfoam based models were used by Wu et al. [12]. Recently, Iasiello et al. [13] compared Nusselt numbers and pressure drops for tomography-based and ideal Kelvin-based geometries. They concluded that tomography-based models tend to be more accurate than Kelvin-based ones. Indeed, a foam model that fits both ideal and tomography-based geometry results is still a challenging task, because of the complex microstructure of a foam.

Transport phenomena in a foam are highly affected by its microstructure. Using Kelvin and Weaire-Phelan ideal foam models, Cunsolo et al. [14] found that radiative characteristics of a foam are strongly dependent on struts shape of the generated foam. Differences larger than $10 \%$ among extinction coefficients were found. The authors concluded that the closer the shape of the ideal foam strut shape to that of a real foam the better the accuracy of the predicted radiative characteristics. Such accuracy is also strongly affected by the foam porosity [15]. The effect of the morphology of Kelvin-foam cell on effective thermal conductivity and convection heat transfer were studied by Pusterla et al. $[16,17]$. The influence of the cell inclination angle, the cell radius at the junction and the strut tapering on effective thermal conductivity was highlighted in [16]. Their effect on pressure drop and heat transfer efficiency was shown in [17]. The heat transfer efficiency accounted for the combined effects of convection heat transfer and pressure drop. Recently, Yang et al. [18] developed an analytical model for the effective thermal conductivity of high porosity open-cell metal foams. A poor effect of strut shape on the effective thermal conductivity was exhibited.

Metal foam commercial-manufacturers, such as ERG Aerospace, use standard techniques to produce foams with assigned PPI and porosity, which affect the strut shape [19]. However, the foam morphology can be modified during the foaming process of the polymeric templates used for replication [17, 20]. Ortona et al. [21] proposed a method to build up foams of any shape by using $3 \mathrm{D}$ printing. This means that the foam morphology that optimizes certain characteristics, like heat transfer and pressure drop, can be designed in the future. So far the effect of strut shape on 
convection heat transfer and pressure drop has not been fully accounted for. As a consequence, the predicted performance of foams have often been affected by marked inaccuracy.

A numerical analysis of the strut shape effect, both on real and on ideal structures, is presented in this paper. The 3D morphology of three real foam samples is scanned with X-ray Computed Tomography (XCT). Foams have equal pores per inch (PPI) and three different porosities, which the strut shape will depend on, as discussed later. Numerical simulations are carried out on real 3D foam structure imaged by XCT data for the evaluation of convection heat transfer coefficients and pressure drops. Results are compared with those obtained for ideal foams based on Lord Kelvin foam structures. Finally, the struts shape that optimizes convection heat transfer and pressure drop in an open-cell metal foam structure is discussed.

\section{Geometry of the foams}

\subsection{Real foams}

\subsubsection{Tomography scans}

$\mathrm{X}$-ray Computed Tomography plays an important role in engineering applications. In fact, it is very useful when the 3-D structure of complex geometries, like open-cell foams, need to be characterized for computational purposes. Three ERG Aerospace cylindrical aluminum-based (Al6101-T6) 40 PPI metal foams, with $10 \mathrm{~mm}$ length, $10 \mathrm{~mm}$ diameter and 0.88, 0.94 and 0.97 porosities, respectively, were analyzed. X-ray Computed Tomography was carried out with an Xradia MicroXCT 400 scanner. Since this paper focuses on strut shape of foams, highresolution scans were employed in order to better highlight the strut shape, using both $1 \mathrm{x}$ and $4 \mathrm{x}$ objective lens. Voxel sizes of the scans were $18 \mu \mathrm{m}$ for the $1 \mathrm{x}$ lens and $5 \mu \mathrm{m}$ for the $4 \mathrm{x}$ lens, respectively. Scans of the same section of the 40 PPI and 0.94 porosity foam are reported in Fig. 1. Xradia's filtered back-projection software was used to reconstruct $\mathrm{x}$-ray projection images [22]. One can clearly remark that struts are crisp in both images.

Images were processed with the freeware program ImageJ. A $7.1 \cdot 7.1 \cdot 10.0 \mathrm{~mm}^{3}$ subvolume of the tomography data was selected, in order to remove non-interesting regions from the corners of the images. Solid and fluid phases in the foam can be clearly distinguished by converting the gray-scale CT data into binary format, using an iterative procedure based on the isodata algorithm [23] to obtain the gray level threshold. The correctness of the threshold calculation was checked using the Otsu method [24] on the same gray-scale CT data. Negligible differences in porosity, no larger than $0.3 \%$, were found.

\subsubsection{Foam microstructure}

Comprehensive studies of foam morphology have been presented in $[19,25]$. The morphology of the three scanned samples is analyzed in the following; the morphological parameters are sketched in Fig. 2. 
A cell is defined as a small compartment and a cluster of cells is defined as a group of them packed in such a way to fill a three-dimensional space [26]. If the cells communicate with each other via open faces, the foam is open-celled and faces are called pores. The solid part of the foam is the strut, which meet in the junctions (Plateau borders).

Diameters can be defined for each of the aforementioned foam morphological characteristics: cell diameter, $d_{c}$; pore diameter, $d_{p}$; strut diameter, $d_{s}$; junction diameter, $d_{j}$. Since the section of the struts is variable, the strut diameter, $d_{s}$, is referred to its middle cross section [21], where it attains its minimum value. Two definitions can be given for each of the above mentioned diameters. A diameter can be defined as the diameter of the largest sphere inscribed into each foam morphological characteristic (definition \#1). Alternatively, as proposed by Inayat et al. [19] and Yu et al. [27], reference can be made to an equivalent circular diameter, $\sqrt{4 \Omega / \pi}$, where $\Omega$ is the area of the cross-section of each measured morphological characteristic (definition \#2). All the above mentioned parameters were obtained using ImageJ on $2 \mathrm{D}$ slices of the $\mathrm{CT}$ data.

Another morphological parameter that gives insight into in transport phenomena is the ratio of the solid/fluid heat transfer surface area (the surface areas of the strut and the junctions), $S_{s+j}$, to the total volume ratio of the foam, $V$, that is $S_{s+j} / V$. It should be remarked that the evaluation of $S_{s+j} / V$ is still a challenging task [19], thus its accuracy may be less than the other parameters mentioned. In this paper it was calculated by using the 2-point correlation function.

A comparison between $1 \mathrm{x}$ and $4 \mathrm{x}$ scans was carried out for all the samples and for the same section, in terms of $d_{c}, d_{p}$ and $S_{s+j} / V$, since they could affect transport coefficients. Differences no larger than $0.3 \%$ in $d_{c}$ and $d_{p}$, and less than $5 \%$ in $S_{s+j} / V$ were found. Therefore, numerical simulations were carried out using $1 \mathrm{x}$ scans.

For diameters based on both the above mentioned definitions, 30 runs for $d_{p}, d_{s}$ and $d_{j}$, and 10 runs for $d_{c}$ were carried out on 2D slices of the CT data, and their average values were then calculated. The distribution of the pore diameters for both definitions is reported in Fig. 3. The figure shows that the distributions gather around a common value, especially when reference is made to the second definition of the pore diameter $\left(d_{p}=\sqrt{4 \Omega / \pi}\right)$.

In addition to the diameters, three further morphological characteristics have been considered in the present study. The first is the length of a strut, $L_{s}$, which is the distance between two junctions. Ten runs were carried out in $L_{s}$ measurements at each porosity. The second is the strut tapering factor, $t$, defined as [17]

$t=d_{j} / d_{s}$ 
The third is a new parameter introduced in the present paper, the foam shape factor, $\beta$, defined as

$\beta=\Omega_{s} / S_{\text {circle }}$

where $\Omega_{s}$ is the average area of the strut cross section and $S_{\text {circle }}$ is the average area of the circle that contains the cross section of the strut. The strut tapering factor and the foam shape factor were evaluated with reference to the foam parameters reported in Fig. 4. The same way as all other morphological characteristics also $S_{\text {circle }}$ is measured using ImageJ on 2D slices of the CT data.

The average values of measured morphological characteristics, with standard deviations, are reported in Table 1. The cell diameter decreases with porosity, since convex struts occupy more room inside a cell, at equal PPI. Figure 3 shows that the pore diameter is almost uniform. We can also notice that, differently from the length of the strut, the cell diameter increases when the porosity decreases. Strut tapering increases, since the increase in the junction diameter is larger than that in the strut diameter. It is also worth noticing that measured porosities are similar to those provided by the manufacturer. Apart from the average pore diameter, $\bar{d}_{p}$, differences in the values obtained making reference to definitions \#1 and \#2 are always less than 1\%. Differences in the pore diameters no larger than $10 \%$ were found. We observed that in an open cell foam deviations in the measured pore diameter are larger than those in other morphological characteristics. Indeed, the standard deviation in pore diameter distribution, reported in Figs. 3a and $3 \mathrm{~b}$, is in the range $0.19-0.23 \mathrm{~mm}$.

\subsubsection{Representative Volume Element (RVE)}

In order to use computational resources effectively, the Representative Volume Element (RVE) approach was adopted in the present study. The RVE approach is very useful when transport in porous media is investigated, because of their complex geometry, that may require for computations a large number of elements. By extracting a volume from the porous medium with the same characteristics of the whole porous medium, named the Representative Volume Element (RVE), allows to save computational resources. Simulations were carried out on a CT data sub-volume whose porosity, $\varepsilon$, and surface to volume ratio, $S_{s+j} / V$, were as close as possible to the corresponding values of the whole foam. The procedure makes the RVE independent of its position in the porous domain. It was detailed in [13] and is outlined in the following. Each CT data is subdivided into cubes with $L_{R V E}$ long sides. The 2-point correlation function is applied on each cube, obtaining a distribution (with a mean and a standard deviation value) for porosity and surface to volume ratio. Observing the strut lengths obtained iterating the process, 
one can remark that the larger the strut length the smaller the standard deviation. Let the coefficient of variation, $C V$, the ratio of the standard deviation to the mean, with the $L_{R V E}$ statistically chosen in the present work its values for the porosity and the surface to volume ratio are less than $15 \%$, for all real foams. The characteristics of the chosen RVEs are resumed in Table 2.

\subsection{Ideal foams}

Finding the structure that best reproduces a foam is a vexed question. The structure has to be space-filling, with a minimum surface area per unit volume. Plateau [28] proposed a rhombic dodecahedron cell shape. Kelvin [29] proposed a tetrakaidecahedron with slightly curved faces: six flat quadrilateral faces and eight hexagonal faces. Nearly a century later, Weaire and Phelan [30] proposed a unit cell made up of two kinds of cells, with a total of eight equal volume cells, whose surface area per unit volume is about $0.3 \%$ smaller than that of the cell proposed by Kelvin. The Kelvin's ideal foam structure has been considered in this study, since it is easier to build up and implies a lighter numerical procedure than the Weaire-Phelan model. Convection heat transfer and pressure drop predicted by the two above said models were compared by Cunsolo et al. [31]; differences were found to be negligible, especially at high porosities.

The Kelvin's foam was generated with Surface Evolver, a program for the study of surfaces shaped by surface tension [32]. A dry foam was first generated; then some iterative steps further reduce the surface energy, using a constrained tapering factor gradient-descent method. The dry foam was characterized by a very high porosity. A fluid phase was added at the Plateau border, generating a wet foam. Further iterations were performed in order to obtain the desired porosities and minimum surface energy. Kelvin foams with different porosities, generated with Surface Evolver, are sketched in Fig. 5. It is shown that the solid matrix becomes thinner when the porosity increases. Since the generated foam is a single-cell ideal foam, that can be considered as extracted from an ensemble of a cluster of many ideal cells, it is a RVE of a macroscopic porous domain made up by ideal Kelvin's cells."

Finally, the generated file was exported as an .off file into MATLAB, in order to obtain Kelvin structures with different strut shapes. Indeed, Surface Evolver generates concave struts for every porosity value by default. Concave, triangular and convex orthogonal cross sections were obtained using a MATLAB/Octave-based mesh generation toolbox called iso2mesh, for each porosity, for a total of 9 structures, as depicted in Fig. 6, where strut shapes of real foams are also reported. Black frames denote the ideal strut that better reproduces the corresponding real foam strut, at each porosity.

Comparisons between ideal and real models are made in terms of equal cell diameter, $d_{c}$, with values reported in Table 1 This means that both ideal and real models are RVE's of macroscopic foams with the same porosity and cell diameter. Ideal models were scaled directly 
with iso2mesh. The surface to volume ratios of the investigated foams are reported in Table 3 . Finally, a bounding box was placed around the domain, with two fictitious inlet and outlet regions, $L_{R V E} / 3$ long, to minimize entrance effects.

\section{Mathematical model}

\subsection{Governing equations}

Since the aim of this paper is to analyze convection heat transfer and pressure drop, only the fluid phase of the foam, air, was taken into account. The maximum value of the pore Reynolds number in the present study is nearly 100, therefore, the steady laminar flow assumption is made $[12,13]$. Since Mach numbers are always lower than 0.3 and temperature variations in the computational domain are as small as to assume air thermophysical properties uniform, the incompressible flow assumption is done. Governing equations for mass, momentum and energy are

$\nabla \cdot u=0$

$\rho \boldsymbol{u} \cdot \nabla \boldsymbol{u}=-\nabla p+\mu \nabla^{2} \boldsymbol{u}$

$\boldsymbol{u} \cdot \nabla T=\alpha \nabla^{2} T$

where $\boldsymbol{u}$ is the velocity vector, $\rho$ the density, $p$ the pressure, $\mu$ the dynamic viscosity, $\alpha$ the thermal diffusivity and $T$ the temperature of the air.

\subsection{Boundary conditions}

Boundary conditions for the real structure are reported in Fig. 7, with the corresponding coordinate systems, for the section $z=0$. Symmetry conditions depicted in Fig. 7 are also valid for planes $y= \pm L_{R V E}$, while inlet and outlet sections boundary conditions are also valid for planes $x=0$ and $x=L_{d o m}$, respectively. Symmetry boundary conditions are imposed in planes $z$ $= \pm L_{R V E} / 2$. For the ideal structure, boundary conditions are the same and, since geometry and boundary conditions are symmetrical with respect to $x y$ and $x z$ planes, only a quarter of the structure was studied.

\subsection{Numerical modelling}

Equations (3-5) with the associated boundary conditions were solved with the Galerkin least square finite element method, using the commercial software COMSOL Multiphysics ${ }^{\circledR}$. A tethraedral mesh was set up using iso2mesh, for both real and ideal models. Size and shape of 
the elements were quasi-uniform, ensuring a high resolution at the boundaries. About two hundred thousand elements were chosen. The grid independence was checked using the Richardson extrapolation and a deviation less than $3 \%$ from the extrapolated values of the differences between the average temperatures of the solid and the fluid and of the pressure drops was found. A convergence criterion of $10^{-4}$ was assumed for the simulations: no significant variation occurred for lower values. Mass and energy balance were checked with an error always less than $1 \%$.

\section{Results and discussion}

\subsection{Data reduction}

Simulations were carried out by assuming three inlet velocities of the air $\left|\boldsymbol{u}_{0}\right|=0.5,1.0$ and $1.5 \mathrm{~m} / \mathrm{s}$, an inlet temperature of the air $T_{\text {in }}=293 \mathrm{~K}$ and a boundary heat flux $|\boldsymbol{q}|=1000 \mathrm{~W} / \mathrm{m}^{2}$. They implied an outlet temperature of the air no larger than $313 \mathrm{~K}$, and, therefore, variations in its temperatures small enough to confirm the validity of the assumptions which the proposed thermo-fluidynamic model was based on. The convection heat transfer coefficient, $h_{c}$, was evaluated by the correlation

$|\boldsymbol{q}|=h_{c}\left(\bar{T}_{s}-\bar{T}_{f}\right)$

where $\bar{T}_{S}$ is the average solid/fluid interfacial surface temperature and $\bar{T}_{f}$ is the volumetric average fluid temperature. With reference to the Volume Averaging Technique (VAT), a volumetric convection heat transfer coefficient, $h_{v}$, was defined as $h_{v}=h_{c}\left(S_{s+j} / V\right)$.

In order to present results in a dimensionless form, a characteristic length had to be defined [33]. Since the surface to volume ratio, $S_{s+j} / N$, was known, the hydraulic diameter, $d_{h}=$ $4 \varepsilon /\left(S_{s+j} / V\right)$, was used as the characteristic length in this paper.

The Nusselt number and Reynolds number were defined as

$$
\begin{aligned}
& \mathrm{Nu}_{\mathrm{h}}=\frac{h_{c} \cdot d_{h}}{\lambda}=\frac{h_{c} 4 \varepsilon\left(S_{s+j} / V\right)}{\lambda} \\
& \operatorname{Re}_{\mathrm{h}}=\frac{\left|\boldsymbol{u}_{0}\right| d_{h}}{v}=\frac{\left|\boldsymbol{u}_{0}\right| 4 \varepsilon\left(S_{s+j} / V\right)}{v}
\end{aligned}
$$

where the thermal conductivity, $\lambda$, and the thermal diffusivity, $\alpha$, of the air were evaluated at the arithmetic average temperature between $\bar{T}_{S}$ and $\bar{T}_{f}$. The same reference temperature was used to evaluate the kinematic viscosity, $v$, in the Reynolds number. 
As far as the pressure drops was concerned, the average pressure was evaluated after simulations in the inlet and in the outlet regions of the computational domain.

\subsection{Results for the real foams}

The convection heat transfer coefficient and volumetric heat transfer coefficient as a function of the velocity, for different porosities of the real foams, are reported in Fig. 8. One can notice that, for the investigated inlet velocities, the convection heat transfer coefficient slightly decreases with the porosity, because of the decrease in the pore velocity. The volumetric convection heat transfer coefficient rapidly decreases with porosity, since surface to volume ratio and heat transfer coefficient decrease at increasing porosity.

Pressure drop per unit length as a function of the velocity, for different porosities of real foams, is presented in Fig. 9. An increase in the porosity slightly reduces pressure drops, because of the lower fraction of solid material in the porous medium. For all the investigated porosities, the dependence law on the velocity is quadratic, in agreement with DarcyForchheimer law

$\frac{\Delta p}{L_{d o m}}=\frac{\mu}{K}\left|\boldsymbol{u}_{0}\right|+\rho C\left|\boldsymbol{u}_{0}\right|^{2}$

where $K$ is the permeability and $C$ is the form coefficient.

It is important to remark that there is a lack of experimental data on convection heat transfer coefficients in literature. The values of the convection heat transfer coefficient, $h_{c}$, and the ratio of the pressure drop per unit length to the Darcian velocity, $\Delta p / L_{d o m}\left|\boldsymbol{u}_{\boldsymbol{o}}\right|$, predicted in the present paper, for $\varepsilon=0.94$, are compared, in Figs.10a and 10b, with correlations presented by Mancin et al. [6], numerical as predictions by Diani et al. [10] and experimental results reported by Bhattacarya et al. [34] and Dukhan [35], for 40 PPI and porosities close to 0.94. A very good agreement is exhibited for both $h_{c}$ and $\Delta p / L_{d o m}\left|\boldsymbol{u}_{\boldsymbol{\theta}}\right|$, indicating that the current model accurately predicts this system.

\subsection{Results for the ideal foams}

Results for ideal foams, with different strut shapes, were derived and compared with results for real foams. At equal values of the porosity, the cell was scaled in order to have a cell diameter equal to that of the real foam, reported in Table 1. The Nusselt number as a function of the Reynolds number, for real and ideal foams, different strut shapes and porosities equal to 0.87 , 0.94 and 0.96, is reported in Figs. 11, 12 and 13, respectively. For all porosities and Reynolds numbers, the agreement between the Nusselt number of the real foam and the ideal foam with the same strut shape as that of the real foam (see Fig. 6) is good. This means that making the 
strut shape closer to that of the real foam improves the accuracy of predictions given by the ideal model. Considering only the ideal foams, a convex strut shape maximizes convection heat transfer. Comparing the convex shape with the concave one, it seems that, when the strut is convex, local flow separation effects are reduced, increasing the convection heat transfer.

The cross section average value of the pressure, referenced to the ambient pressure, as a function of the velocity of the air, for real and ideal foams, different strut shapes and porosities equal to 0.87, 0.94 and 0.96, is reported in Figs. 14, 15 and 16, respectively. All figures point out large differences in the pressure values in the inlet section of the foam $(x=0)$ for ideal as well as real foams. A comparison among the ideal foams shows that at the higher investigated porosity the pressure field is practically unaffected by the foam shape factor. At lower porosities the maximum pressure is exhibited when the struts shape is concave $(\beta=0.49)$, since it maximizes drag forces [5, 34]. Pressure is quite independent of the strut shape when it is triangular $(\beta=0.59)$ or convex $(\beta=0.67)$.

If the comparison is now made between the real and the ideal foams, one can remark that the more accurate prediction of the pressure field is obtained when the ideal foam shape factor is the same as that of the real foam, at any porosity and velocity. It is worth remarking that immediately downstream of the inlet section differences in the pressure field between real and ideal foams sharply decrease while ideal foams strongly over-predict the pressure in it. Larger differences between pressures in the inlet section occur since the localized pressure drop depends on the struts arrangement that, in turn, depends on the RVE choice of the real foam.

The pressure field referenced by the ambient pressure, in the inlet cross section, for a triangular shape of the strut $(\beta=0.49), 1.5 \mathrm{~m} / \mathrm{s}$ velocity of the air, and 0.94 porosity, predicted for real and ideal foams, is reported in Figs. 17a and 17b, respectively. The marked difference between the predicted pressure fields in Fig.17a and $17 \mathrm{~b}$ is the result of the difference in foam morphology at the inlet section, where the free area in the real foam is lower than that in the ideal foam based on Kelvin's model.

\section{Conclusions}

The scarcely investigated effect of struts shape on convection heat transfer and pressure in open-cell metal foams has been analyzed numerically by introducing a parameter that characterizes the shape of the strut. After a morphological analysis of real foams samples, simulations have been carried out on Kelvin's ideal foam model and on tomography-based real foams.

As far as real foam models are concerned, it has been shown that the convection heat transfer coefficient slightly decreases with porosity, while the volumetric convection heat transfer coefficient markedly decreases. Pressure drop increases when porosity lowers. Comparisons with ideal models have been carried out. Nusselt numbers for real foams are in good agreement 
with those for ideal ones when the strut shape of the ideal model is accurately accounted for. As to the pressure, apart from the inlet section of the foam, the predictions of real models are in good agreement with the real model, provided the foam shape factor be the same.

Finally, it is worth remarking that the convex strut shape maximizes the convection heat transfer and minimizes the pressure drops, for any porosity and velocity.

\section{Acknowledgements}

Authors thank the Università di Napoli Federico II for funding this study within the agreement with the University of Connecticut and the Smart grid con sistemi di poligenerazione distribuita (Poligrid). WKSC gratefully acknowledges financial support from the National Science Foundation (Award CBET-1134052) and an Energy Frontier Research Center on Science-Based Nano-Structure Design and Synthesis of Heterogeneous Functional Materials for Energy Systems (Heterofoam Center) funded by the US Department of Energy, Office of Science, Office of Basic Energy Sciences (Award DE-SC0001061).

\section{References}

[1] S. Mahjoob, K. Vafai, A synthesis of fluid and thermal transport models for metal foam heat exchangers, International Journal of Heat and Mass Transfer 51 (15) (2008) 3701-3711.

[2] X. Chen, F. Tavakkoli, K. Vafai, Analysis and characterization of metal foam-filled doublepipe heat exchangers, Numerical Heat Transfer, Part A: Applications 68 (10) (2015) 1031-1049.

[3] A. Amiri, K. Vafai, Analysis of dispersion effects and non-thermal equilibrium, nonDarcian, variable porosity incompressible flow through porous media, International Journal of Heat and Mass Transfer 37 (6) (1994) 939-954.

[4] S. Mancin, C. Zilio, A. Cavallini, L. Rossetto, Heat transfer during air flow in aluminum foams, International Journal of Heat and Mass Transfer 53 (21-22) (2010) 4976-4984.

[5] S. Mancin, C. Zilio, A. Cavallini, L. Rossetto, Pressure drops during air flow in aluminum foams, International Journal of Heat and Mass Transfer 53 (15-16) (2010) 3121-3130.

[6] S. Mancin, C. Zilio, A. Diani, L. Rossetto, Air forced convection through metal foams: Experimental results and modeling, International Journal of Heat and Mass Transfer 62 (2013) $112-123$.

[7] S. Haussener, P. Coray, W. Lipinski, P. Wyss, A. Steinfeld, Tomography-based heat and mass transfer characterization of reticulate porous ceramics for high-temperature processing, Journal of Heat Transfer 132 (2) (2010) 023305-1-9.

[8] P. Ranut, E. Nobile, L. Mancini, High resolution microtomography-based CFD simulation of flow and heat transfer in aluminium metal foams, Applied Thermal Engineering 69 (2014) 230-240. 
[9] M. Zafari, M. Panjepour, M.D. Emami, M. Meratian, Microtomography-based numerical simulation of fluid flow and heat transfer in open cell metal foams, Applied Thermal Engineering, 80 (2015) 347-354.

[10] A. Diani, K. K. Bodla, L. Rossetto, S. V. Garimella, Numerical investigation of pressure drops and heat transfer through reconstructed metal foams and comparison against experiments, International Journal of Heat and Mass Transfer 88 (2015) 508-515.

[11] T.J. Lu, H.A. Stone, M.F. Ashby, Heat transfer in open-cell metal foams, Acta Materialia 46 (10) (1998) 3619-3635.

[12] Z. Wu, C. Caliot, G. Flamant, Z. Wang, Numerical simulation of convection heat transfer between air flow and ceramic foams to optimize volumetric solar air receiver performances, International Journal of Heat and Mass Transfer 54 (7-8) (2011) 1527-1537.

[13] M. Iasiello, S. Cunsolo, M. Oliviero, W.M. Harris, N. Bianco, W.K.S. Chiu, V. Naso, Numerical Analysis of Heat Transfer and Pressure Drops in Metal Foams for Different Morphological Models, Journal of Heat Transfer 136 (11) (2014) 112601-1-10.

[14] S. Cunsolo, M. Oliviero, W.M. Harris, A. Andreozzi, N. Bianco, W.K.S. Chiu, V. Naso, Monte Carlo determination of radiative properties of metal foams: Comparison between idealized and real cell structures, International Journal of Thermal Sciences 87 (2015) 94-102.

[15] D. Baillis, N. Bianco, S. Cunsolo, V. Naso, M. Oliviero, Effects of ligaments shape on radiative heat transfer in metal foams, in: Proceedings of the Third International Conference on Computational Methods for Thermal Problems, Giannini Editore, Napoli, Italy, 2014, pp. 113116.

[16] S. Pusterla, A. Ortona, C. D'Angelo, M. Barbato, The influence of cell morphology on the effective thermal conductivity of reticulated ceramic foams, Journal of Porous Materials 19 (3) (2012) 307-315.

[17] S. Pusterla, M. Barbato, A. Ortona, C. D'Angelo, Numerical study of cell morphology effects on convection heat transfer in reticulated ceramics, International Journal of Heat and Mass Transfer 55(25-26) (2012) 7902-7910.

[18] X.H. Yang, J.X. Bai, H.B. Yan, J.J. Kuang, T.J. Lu, T. Kim, An analytical unit cell model for the effective thermal conductivity of high porosity open-cell metal foams, Transport in porous media 102 (3) (2014) 403-426.

[19] A. Inayat, H. Freund, T. Zeiser, W. Schwieger, Determining the specific surface area of ceramic foams: The tetrakaidecahedra model revisited, Chemical Engineering Science 66 (6) (2011) 1179-1188.

[20] N.J. Mills, Polymer Foams Handbook: Engineering and biomechanics applications and design guide, Elsevier, 2007.

[21] A. Ortona, C. D’Angelo, S. Gianella, D. Gaia, Cellular ceramics produced by rapid prototyping and replication, Materials Letters 80 (2012) 95-98. 
[22] J.R. Izzo, A.S. Joshi, K.N. Grew, W.K.S. Chiu, A. Tkachuk, S.H. Wang, W. Yun, Nondestructive Reconstruction and analysis of SOFC anodes using X-ray computed tomography at Sub-50 nm resolution, Journal of the Electrochemical Society 155 (5), B504B508.

[23] T.W. Ridler, S. Calvard, Picture thresholding using an iterative selection method, IEEE Transactions on systems, man and cybernetics 8 (8) (1978) 630-632.

[24] N. Otsu, A threshold selection method from gray-level histograms, IEEE Transactions on systems, man and cybernetics 9 (1) (1979) 62-66.

[25] J. Bock, A.M. Jacobi, Geometric classification of open-cell metal foams using X-ray micro-computed tomography 75 (2013) 35-43.

[26] L.J. Gibson and M.F. Ashby, Cellular solids: structure and properties, 2nd edition, Cambridge University Press, Cambridge, UK.

[27] W.M. Yu, S.C. Harlock, G.A. V. Leaf, K.W. Yeung, Morphological properties of flexible polyurethane foams and their relationship with cell count, Research Journal of Textile and Apparel 1 (1) (1997) 18-23.

[28] J.A.F. Plateau, Statique expérimentale et théorique des liquides soumis aux seules forces moléculaires, Gauthier-Villars, Paris, France, 1873.

[29] Lord Kelvin (Sir William Thomson), On the division of space with minimum partitional area, Acta Mathematica 11 (1-4) 121-134.

[30] D. Weaire, R. Phelan, A counter-example to Kelvin's conjecture on minimal surfaces, Philosophical Magazine Letters 69 (2) 107-110.

[31] S. Cunsolo, M. Iasiello, M. Oliviero, N. Bianco, W.K.S. Chiu, V. Naso, Numerical Analysis of Heat Transfer and Pressure Drops in Metal Foams for Different Morphological Models, Journal of Heat Transfer 138 (2) (2014) 022601-1-7.

[32] K.A. Brakke, The Surface Evolver, Experimental Mathematics 1(2) (1992) 141-165.

[33] M. Iasiello, S. Cunsolo, N. Bianco, V. Naso, M. Oliviero, W.M. Harris, W.K.S. Chiu, Forced convection heat transfer in metal foams: the characteristic length issue, in: Proceedings of the Third International Conference on Computational Methods for Thermal Problems, Giannini Editore, Napoli, Italy, 2014, pp. 117-120.

[34] A. Bhattacharya, V.V. Calmidi, R.L. Mahajan, Thermophysical properties of high porosity metal foams, International Journal of Heat and Mass Transfer 45 (5) (2002) 1017-1031.

[35] N. Dukhan, Correlations for the pressure drops for flow through metal foam, Experiments in Fluids 41 (4) (2006) 665-672. 


\section{Captions to the figures}

Fig. 1

Fig. 2

Fig. 3

Fig. 4

Fig. 5

Fig. 6

Fig. 7

Fig. 8

Fig. 9

Fig. 10

Fig. 11

Fig. 12

Fig. 13

Fig. 14

Fig. 15

Fig. 17

$1 \mathrm{x}$ and $4 \mathrm{x}$ scans for a 40 PPI and 0.94 porosity foam.

Foam morphological parameters.

Distribution of the pore diameter: a) definition \#1; b) definition \#2.

Foam parameters used in the evaluation of the strut tapering and the foam shape factors.

Kelvin foams generated with Surface Evolver: a) $\varepsilon=0.96$, b) $\varepsilon=0.94$; c) $\varepsilon=0.87$.

Ideal strut shapes generated using Surface Evolver and cross section of real strut shapes imaged using x-ray microtomography.

Boundary conditions for the real foam.

Convection heat transfer coefficient and volumetric convection heat transfer coefficient $v s$. the velocity, for different porosities of real foams.

Pressure drop per unit length $v s$. the velocity, for different porosities of real foams. Comparisons of predictions for real foams with data taken from the literature, for 40 PPI and $\varepsilon \approx 0.94$ foams: a) convection heat transfer coefficient; b) pressure drops.

Nusselt number vs. Reynolds number, for real and ideal foams, different strut shapes, $\varepsilon=0.87$.

Nusselt number vs. Reynolds number, for real and ideal foams, different strut shapes, $\varepsilon=0.94$.

Nusselt number $v s$. Reynolds number, for real and ideal foams, different strut shapes, $\varepsilon=0.96$.

Cross section average value of the pressure, referred to the ambient pressure, vs. the axial coordinate, for real and ideal foams, different strut shapes and porosities, $\left|\boldsymbol{u}_{\boldsymbol{0}}\right|=0.5 \mathrm{~m} / \mathrm{s}$.

Cross section average value of the pressure, referred to the ambient pressure, vs. the axial coordinate, for real and ideal foams, different strut shapes and porosities, $\left|\boldsymbol{u}_{\boldsymbol{0}}\right|=1.0 \mathrm{~m} / \mathrm{s}$.

Fig. 16 Cross section average value of the pressure, referred to the ambient pressure, vs. the axial coordinate, for real and ideal foams, different strut shapes and porosities, $\left|u_{0}\right|=1.5 \mathrm{~m} / \mathrm{s}$.

Pressure field for $x=0,\left|u_{0}\right|=1.5 \mathrm{~m} / \mathrm{s}, \varepsilon=0.94$ : a) real foam (triangular); b) ideal foam (triangular). 


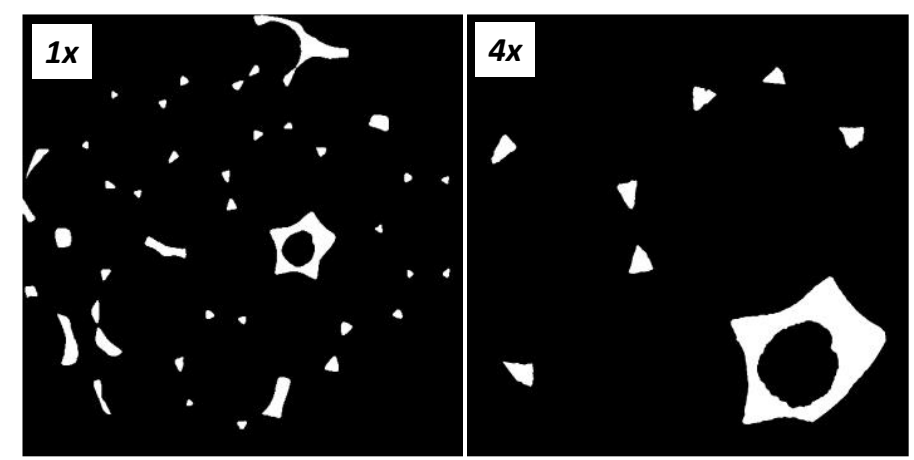

Figure $1-1 x$ and $4 x$ scans for a 40 PPI and 0.94 porosity foam. 


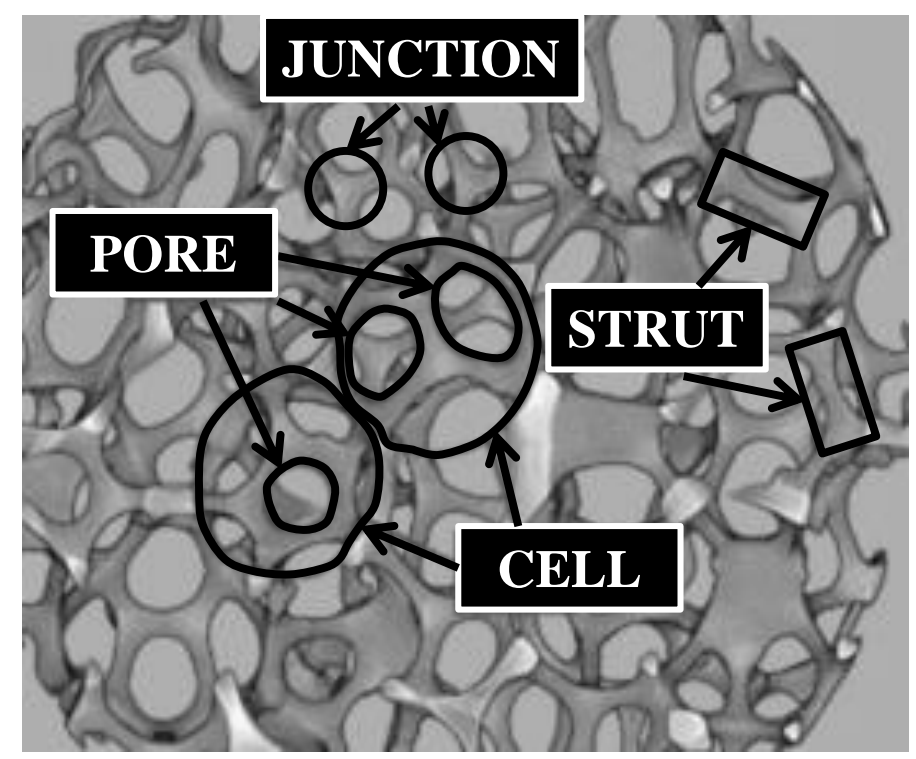

Figure 2 - Foam morphological parameters. 

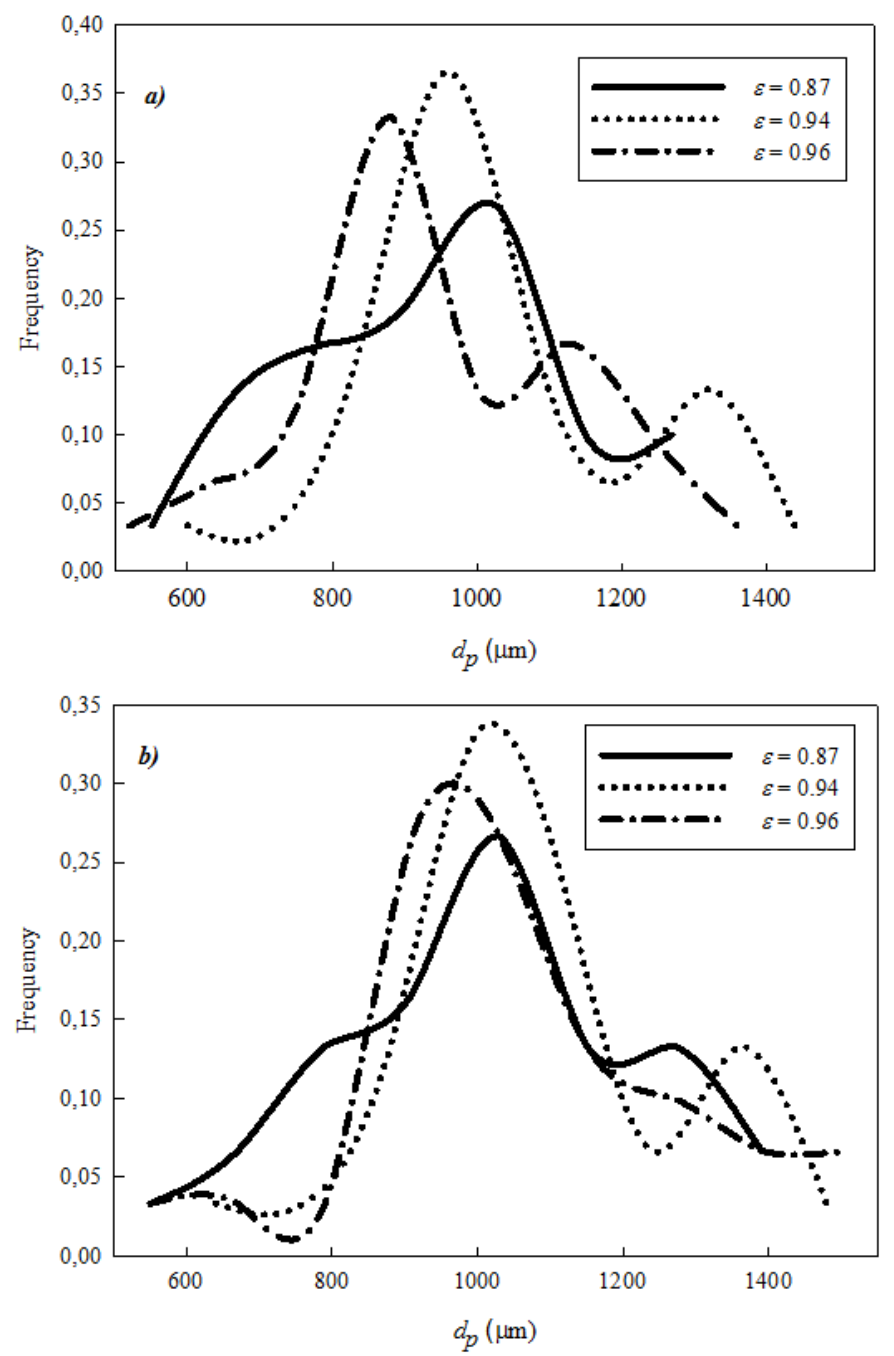

Figure 3 - Distribution of the pore diameter:

a) definition \# 1; b) definition \#2. 


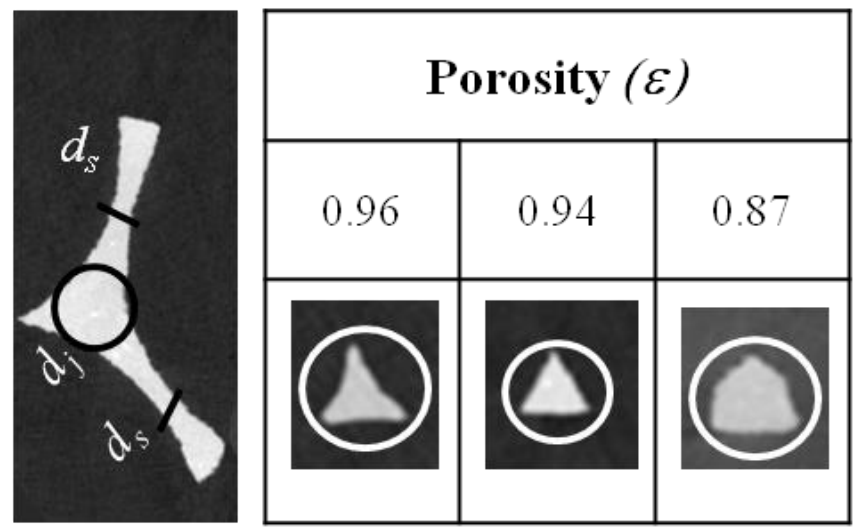

Figure 4 - Foam parameters used in the evaluation of the strut tapering and the foam shape factors. 


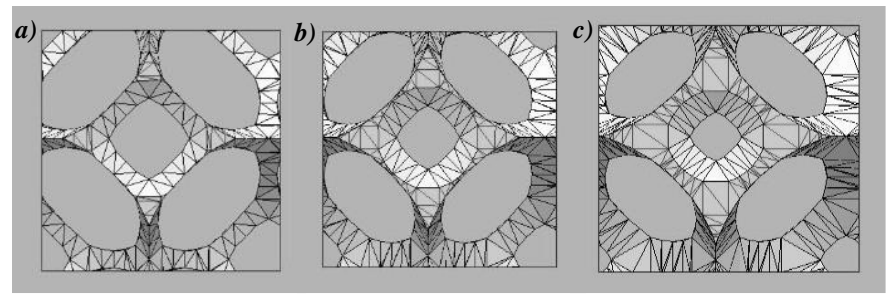

Figure 5 - Kelvin foams generated with Surface Evolver:

a) $\varepsilon=0.96$, b) $\varepsilon=0.94$, c) $\varepsilon=0.87$. 


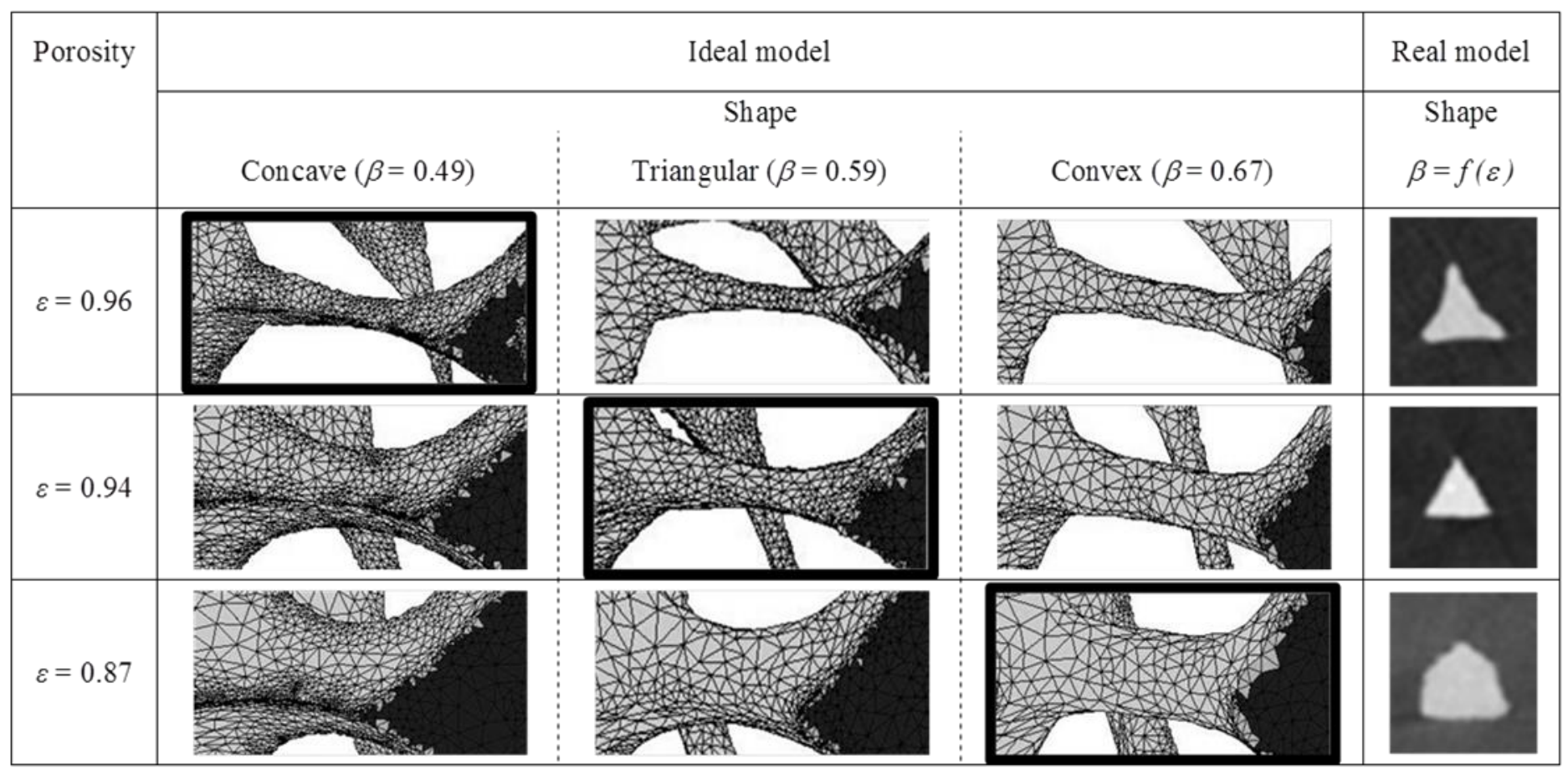

Figure 6 - Ideal strut shapes generated using Surface Evolver and cross section of real strut shapes imaged using X-ray Computed Tomography. 


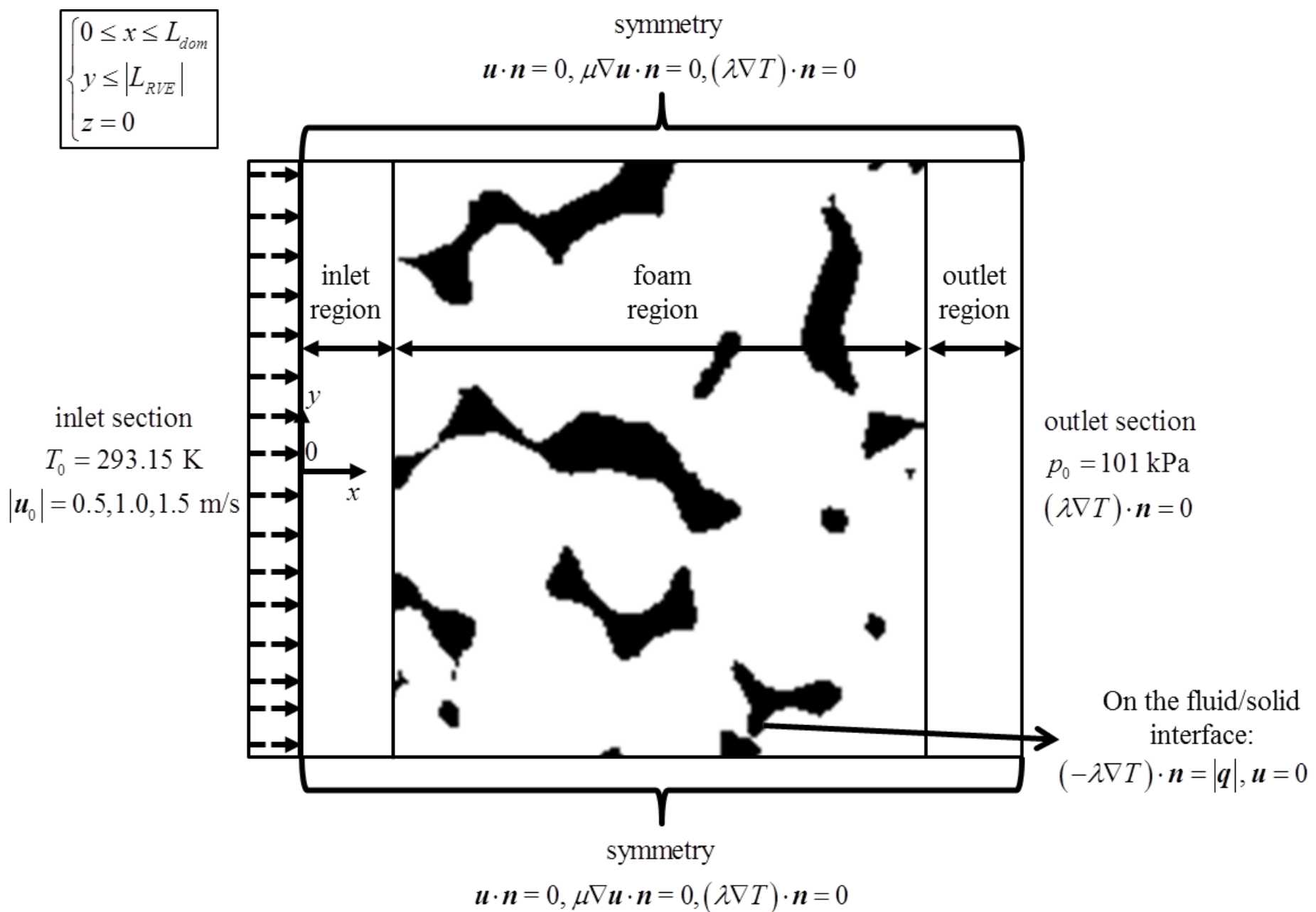

Figure 7 - Boundary conditions for the real foam. 


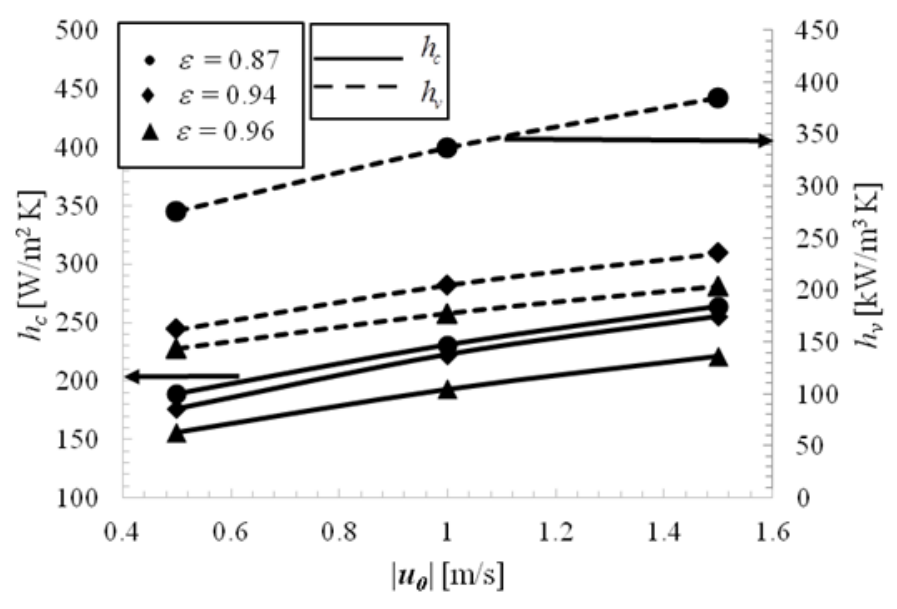

Figure 8 - Convection heat transfer coefficient and volumetric convection heat transfer coefficient $v s$. the velocity, for different porosities of real foams. 


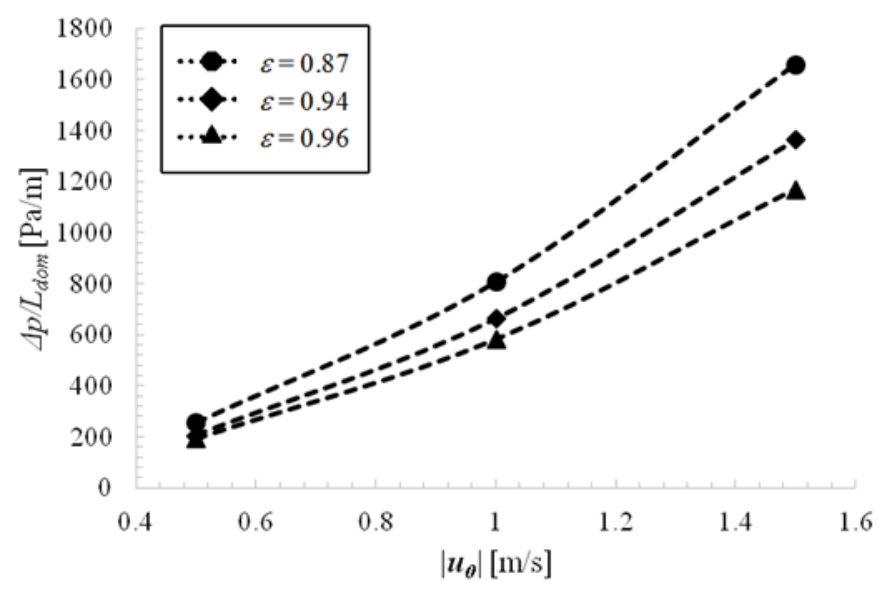

Figure 9 - Pressure drop per unit length $v s$. the velocity, for different porosities of real foams. 

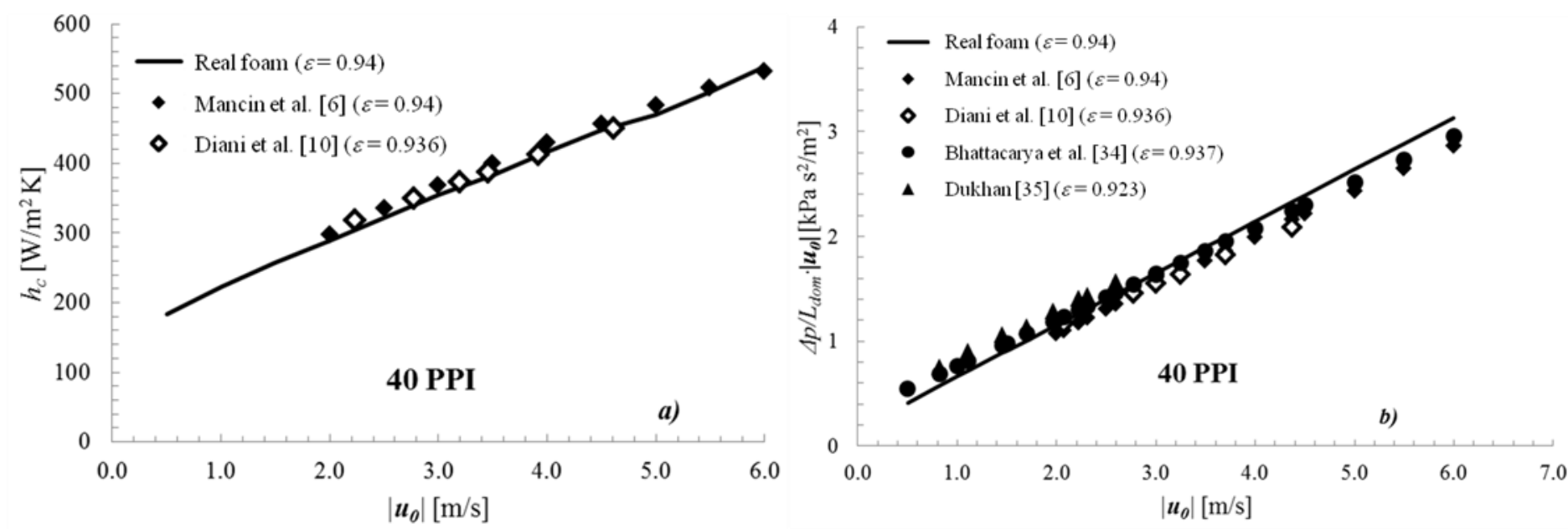

Figure 10 - Comparisons of predictions for real foams with data taken from the literature, for 40 PPI and $\varepsilon \approx 0.94$ foams: a) convection heat transfer coefficient;

b) pressure drop. 


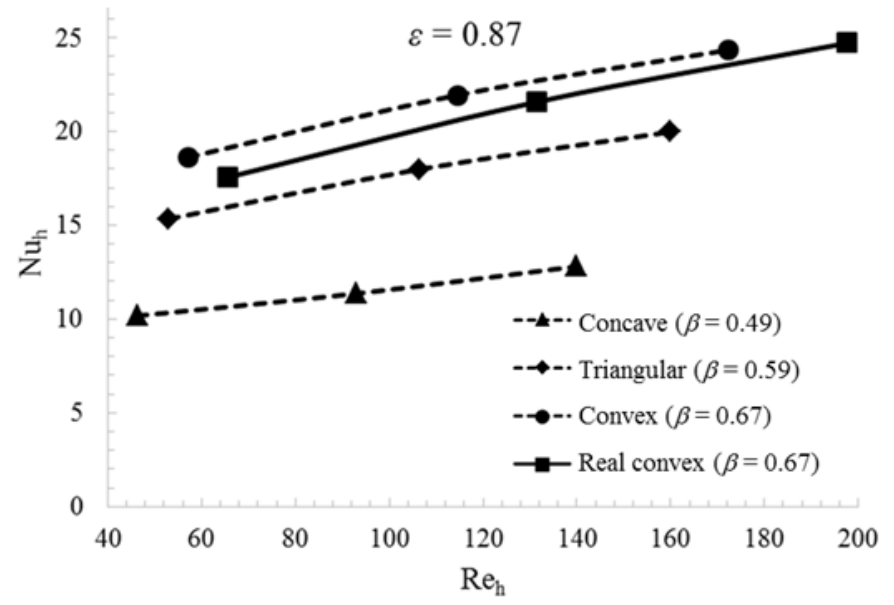

Figure 11 - Nusselt number vs. Reynolds number, for real and ideal foams, different strut shapes, $\varepsilon=0.87$. 


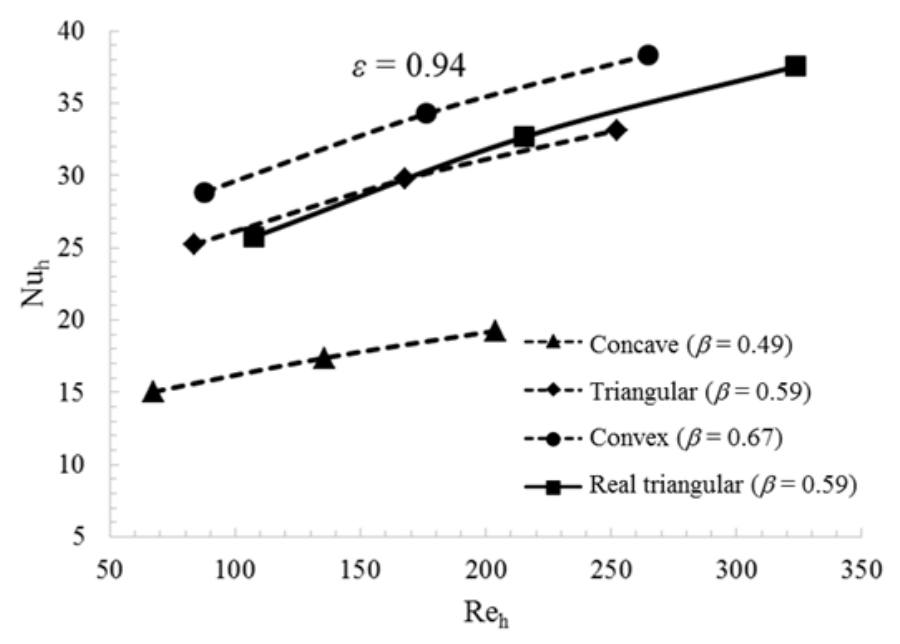

Figure 12 - Nusselt number vs. Reynolds number, for real and ideal foams, different strut shapes, $\varepsilon=0.94$. 


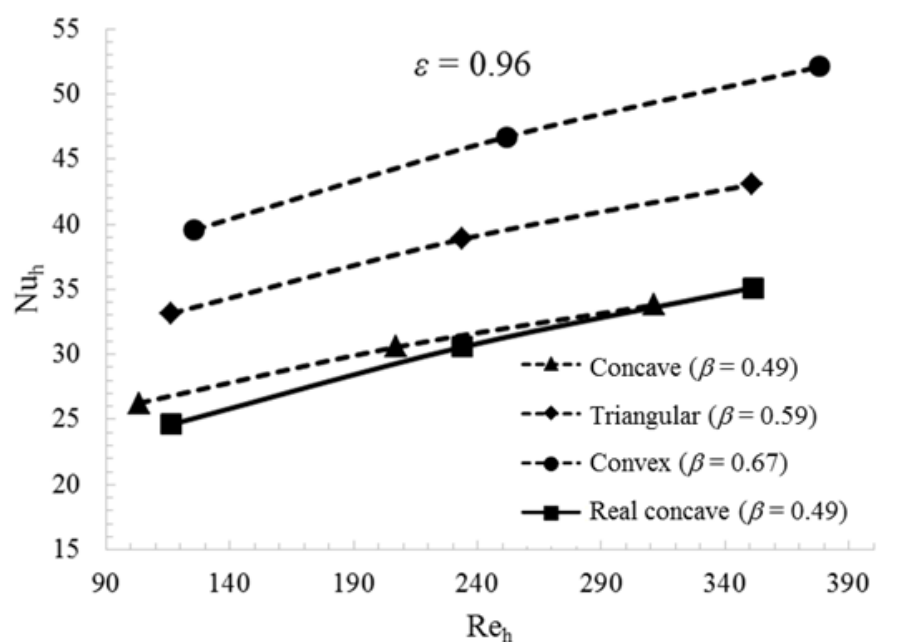

Figure 13 - Nusselt number vs. Reynolds number, for real and ideal foams, different strut shapes, $\varepsilon=0.96$. 

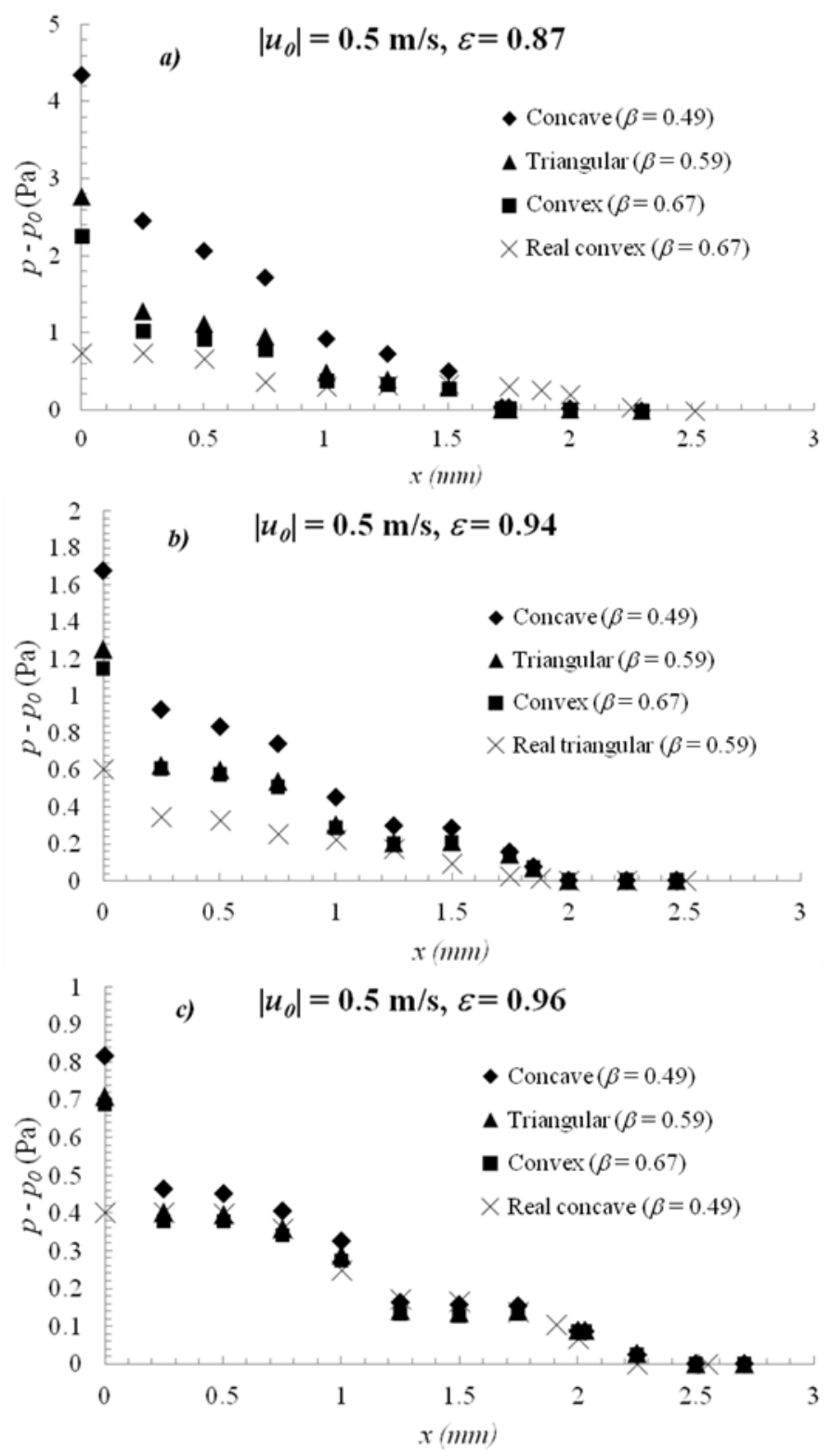

Figure 14 - Cross section average value of the pressure, referred to the ambient pressure, $v s$. the axial coordinate, for real and ideal foams, different strut shapes and porosities, $\left|u_{0}\right|=0.5 \mathrm{~m} / \mathrm{s}$. 

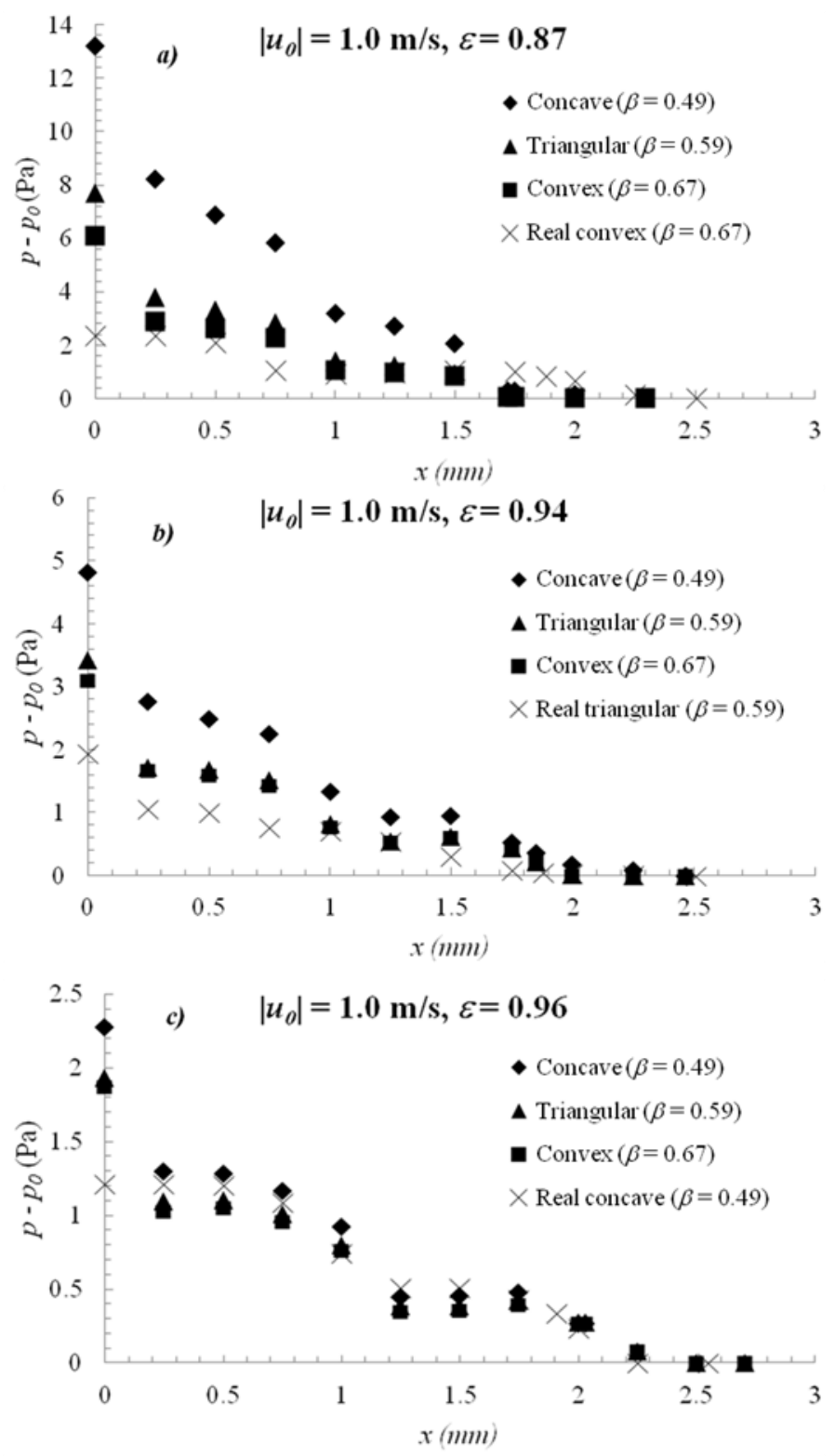

Figure 15 - Cross section average value of the pressure, referred to the ambient pressure, $v s$. the axial coordinate, for real and ideal foams, different strut shapes and porosities, $\left|u_{0}\right|=1.0 \mathrm{~m} / \mathrm{s}$. 

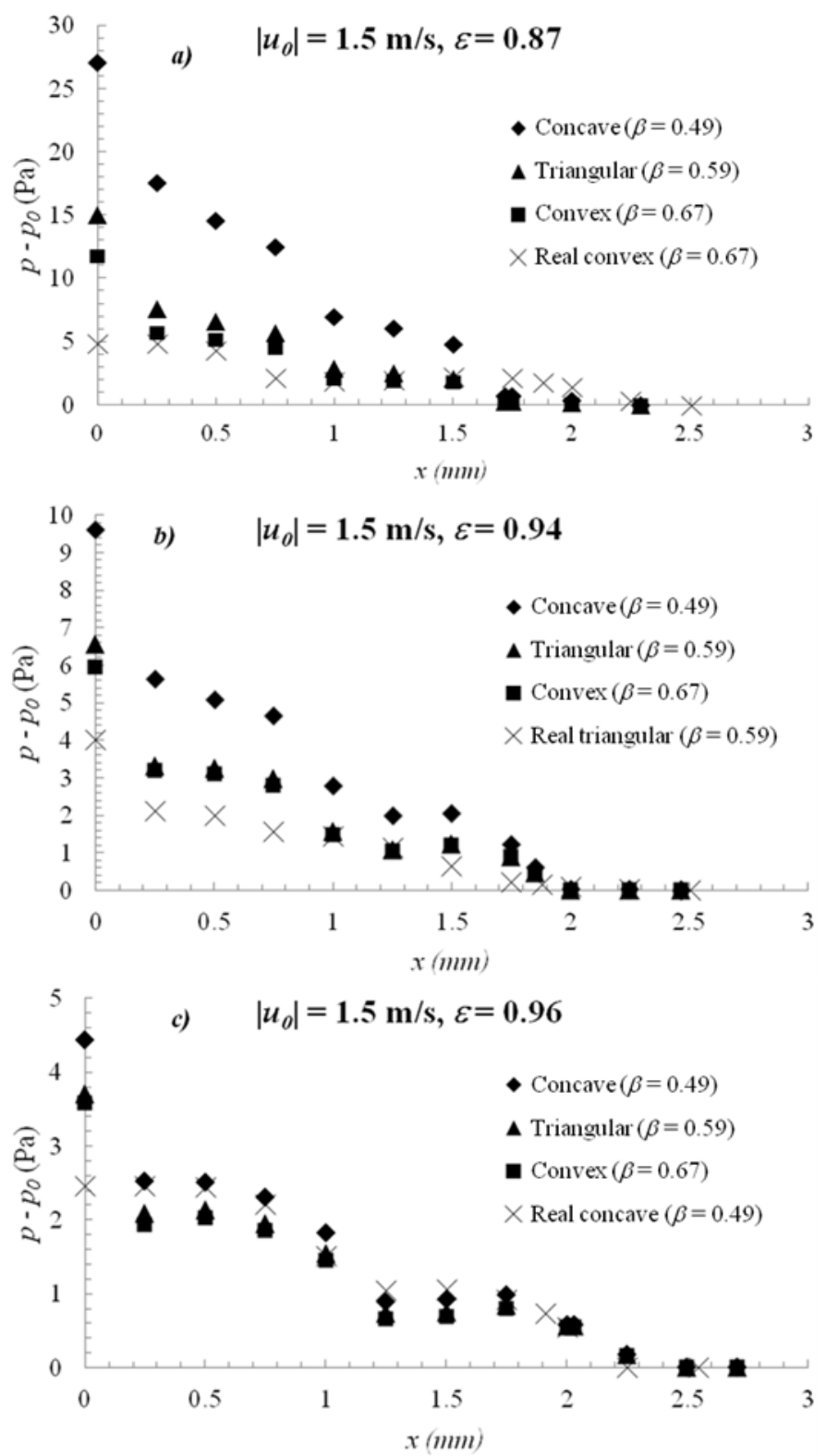

Figure 16 - Cross section average value of the pressure, referred to the ambient pressure, $v s$. the axial coordinate, for real and ideal foams, different strut shapes and porosities, $\left|u_{0}\right|=1.5 \mathrm{~m} / \mathrm{s}$. 
Real foam (triangular), $\beta=0.59$

$$
\left|\boldsymbol{u}_{0}\right|=1.5 \mathrm{~m} / \mathrm{s}, \varepsilon=0.94
$$

$x=0$

$p-p_{0}$

a) $(P a)$

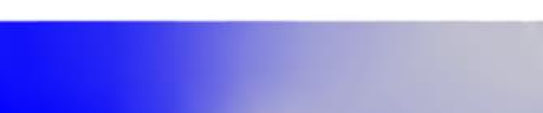

$\Delta 4.3423$
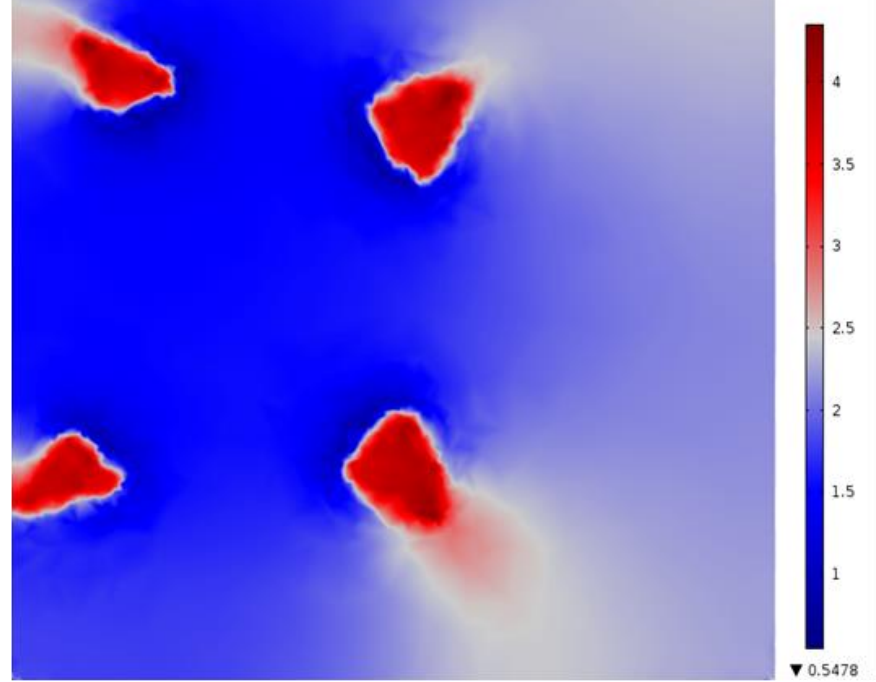

Ideal foam (triangular), $\beta=0.59$

$$
\begin{gathered}
\left|\boldsymbol{u}_{0}\right|=1.5 \mathrm{~m} / \mathrm{s}, \varepsilon=0.94 \\
x=0
\end{gathered}
$$$$
p-p_{0}
$$
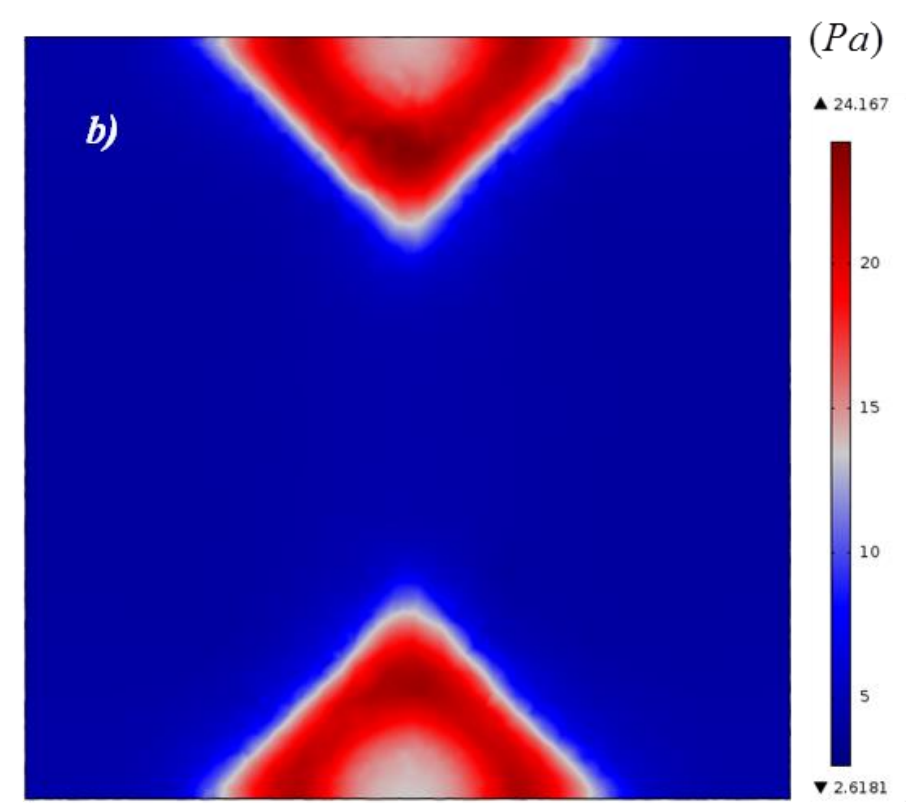

Figure 17 - Pressure field for $x=0,\left|u_{0}\right|=1.5 \mathrm{~m} / \mathrm{s}, \varepsilon=0.94$ :

a) real foam (triangular); b) ideal foam (triangular). 
Table 1. Average values of measured morphological characteristics (standard deviation in parentheses).

\begin{tabular}{|c|c|c|c|c|c|c|c|c|c|c|c|}
\cline { 4 - 11 } \multicolumn{1}{c|}{} & \multicolumn{4}{|c|}{ Definition \#1 } & \multicolumn{4}{c|}{ Definition \#2 } \\
\hline$\varepsilon$ & $\beta$ & $\begin{array}{c}\bar{L}_{s} \\
(\mathrm{~mm})\end{array}$ & $\begin{array}{c}S_{s+j} / V \\
(1 / \mathrm{m})\end{array}$ & $\begin{array}{c}\bar{d}_{c} \\
(\mathrm{~mm})\end{array}$ & $\begin{array}{c}\bar{d}_{p} \\
(\mathrm{~mm})\end{array}$ & $\begin{array}{c}\bar{d}_{s} \\
(\mathrm{~mm})\end{array}$ & $\bar{t}$ & $\begin{array}{c}\bar{d}_{c} \\
(\mathrm{~mm})\end{array}$ & $\begin{array}{c}\bar{d}_{p} \\
(\mathrm{~mm})\end{array}$ & $\begin{array}{c}\bar{d}_{s} \\
(\mathrm{~mm})\end{array}$ & $\bar{t}$ \\
\hline 0.87 & $\begin{array}{c}0.67 \\
(0.07)\end{array}$ & $\begin{array}{c}1.32 \\
(0.15)\end{array}$ & 1408 & $\begin{array}{c}1.72 \\
(0.15)\end{array}$ & $\begin{array}{c}0.88 \\
(0.19)\end{array}$ & $\begin{array}{c}0.29 \\
(0.11)\end{array}$ & $\begin{array}{c}2.2 \\
(0.3)\end{array}$ & $\begin{array}{c}1.71 \\
(0.16)\end{array}$ & $\begin{array}{c}0.99 \\
(0.23)\end{array}$ & $\begin{array}{c}0.29 \\
(0.09)\end{array}$ & $\begin{array}{c}2.1 \\
(0.3)\end{array}$ \\
\hline 0.94 & $\begin{array}{c}0.59 \\
(0.06)\end{array}$ & $\begin{array}{c}1.37 \\
(0.14)\end{array}$ & 954 & $\begin{array}{c}1.85 \\
(0.21)\end{array}$ & $\begin{array}{c}0.95 \\
(0.19)\end{array}$ & $\begin{array}{c}0.20 \\
(0.04)\end{array}$ & $\begin{array}{c}2.1 \\
(0.3)\end{array}$ & $\begin{array}{c}1.84 \\
(0.21)\end{array}$ & $\begin{array}{c}1.00 \\
(0.19)\end{array}$ & $\begin{array}{c}0.18 \\
(0.03)\end{array}$ & $\begin{array}{c}2.0 \\
(0.2)\end{array}$ \\
\hline 0.96 & $\begin{array}{c}0.49 \\
(0.05)\end{array}$ & $\begin{array}{c}1.45 \\
(0.14)\end{array}$ & 922 & $\begin{array}{c}2.03 \\
(0.17)\end{array}$ & $\begin{array}{c}0.89 \\
(0.20)\end{array}$ & $\begin{array}{c}0.16 \\
(0.05)\end{array}$ & $\begin{array}{c}1.8 \\
(0.2)\end{array}$ & $\begin{array}{c}1.99 \\
(0.16)\end{array}$ & $\begin{array}{c}0.96 \\
(0.22)\end{array}$ & $\begin{array}{c}0.15 \\
(0.02)\end{array}$ & $\begin{array}{c}1.5 \\
(0.2)\end{array}$ \\
\hline
\end{tabular}


Table 2. Characteristics of the chosen RVEs.

\begin{tabular}{|c|c:c|c|c|}
\hline $\begin{array}{c}L_{R V E} \\
(\mathrm{~mm})\end{array}$ & $\varepsilon$ & $10^{2} \mathrm{CV}$ & $\begin{array}{c}S_{s+j} / V \\
(1 / \mathrm{m})\end{array}$ & $10^{2} \mathrm{CV}$ \\
\hline 1.88 & 0.87 & 2.47 & 1408 & 13.79 \\
\hline 1.89 & 0.94 & 0.86 & 954 & 7.10 \\
\hline 1.91 & 0.96 & 0.39 & 922 & 3.37 \\
\hline
\end{tabular}


Table 3. Surface to volume ratio values of the investigated foams.

\begin{tabular}{|c|c|c|c|c|c|}
\hline & \multicolumn{4}{|c|}{$S_{s+j} / V(1 / \mathrm{m})$} \\
\hline & & \multicolumn{3}{|c|}{ Ideal (Kelvin) } & \multirow[t]{2}{*}{ Real (XCT) } \\
\hline$d_{c}(\mathrm{~mm})$ & $\varepsilon$ & Concave & Triangular & Convex & \\
\hline 1.72 & 0.96 & 1142 & 945 & 779 & 922 \\
\hline 1.85 & 0.94 & 1509 & 1223 & 1166 & 954 \\
\hline 2.03 & 0.87 & 2053 & 1801 & 1675 & 1408 \\
\hline
\end{tabular}

